Revue d'histoire de l'Amérique française

REVUE D.HISTOIRE DE L'AMÉRIQUE FRANÇAISE

\title{
Les naissances illégitimes sur les rives du Saint-Laurent avant 1730
}

\section{Lyne Paquette et Réal Bates}

Volume 40, numéro 2, automne 1986

URI : https://id.erudit.org/iderudit/304446ar

DOI : https://doi.org/10.7202/304446ar

Aller au sommaire du numéro

Éditeur(s)

Institut d'histoire de l'Amérique française

ISSN

0035-2357 (imprimé)

1492-1383 (numérique)

Découvrir la revue

Citer cette note

Paquette, L. \& Bates, R. (1986). Les naissances illégitimes sur les rives du Saint-Laurent avant 1730. Revue d'histoire de l'Amérique française, 40(2), 239-252. https://doi.org/10.7202/304446ar
Résumé de l'article

La statistique démographique permet de mesurer les naissances illégitimes et les conceptions prénuptiales pour les populations anciennes, offrant ainsi un éclairage nouveau sur leurs moeurs. Les deux contributions qui suivent offrent des résultats inédits concernant ces deux phénomènes dans la vallée du Saint-Laurent d'avant 1730. Si les interdits religieux en matière de sexualité hors mariage ne furent pas toujours respectés, il n'en demeure pas moins que seulement 1,25\% de l'ensemble des naissances sont illégitimes et $6,1 \%$ des premières naissances ont été conçues avant le mariage. Une évolution se dessine cependant dans le temps, de même que des distinctions doivent être faites selon le milieu urbain ou rural. L'état matrimonial des mères ainsi que leur âge sont aussi des facteurs déterminants de la fréquence de ces comportements. Signalons enfin que le mouvement saisonnier de ces conceptions hors mariage, sensiblement différent de celui des conceptions légitimes, et la très forte surmortalité des bâtards ne manquent pas d'intérêt pour l'étude de cette population ancienne.
Tous droits réservés @ Institut d'histoire de l'Amérique française, 1986
Ce document est protégé par la loi sur le droit d'auteur. L’utilisation des services d'Érudit (y compris la reproduction) est assujettie à sa politique d'utilisation que vous pouvez consulter en ligne.

https://apropos.erudit.org/fr/usagers/politique-dutilisation/ 


\title{
LES NAISSANCES ILLÉGITIMES SUR LES RIVES DU SAINT-LAURENT AVANT 1730 ${ }^{1}$
}

\author{
LYNE PAQUETTE \\ REAL BATES \\ Programme de recherche en démographie historique \\ Université de Montréal
}

\section{INTRODUCTION}

Les caractéristiques démographiques de la population canadienne sous le Régime français sont de mieux en mieux connues. La fécondité des premiers colons, notamment, a été remarquablement analysée par Jacques Henripin ${ }^{2}$, puis par Hubert Charbonneau ${ }^{3}$. Cependant, un secteur limité de ce phénomène reste relativement dans l'ombre: les naissances illégitimes.

Les études sur le sujet ont été peu nombreuses jusqu'à maintenant, faute de données adéquates. Le généalogiste Cyprien Tanguay fournit une liste des naissances illégitimes par décennie, mais seulement à partir du début du 18e siècle. Dans le volume IV de son Dictionnaire généalogique, il mentionne seulement 171 naissances illégitimes pour la période 1701-1730, pour les paroisses catholiques de la NouvelleFrance ${ }^{4}$; or nous en avons relevé 565 pour la période $1700-1729$. Il ne fait aucun doute que son décompte est incomplet, pour plusieurs raisons. Il n'a dû vraisemblablement considérer comme illégitimes que les enfants déclarés comme tels dans les registres alors que notre définition en englobe davantage. D'autre part, comme Mgr Tanguay s'intéressait avant tout aux familles, il a probablement fait moins d'efforts dans la collecte des naissances illégitimes. Le phénomène lui paraissant moralement condamnable, il a pu omettre plusieurs de ces naissances. Il ne fait aucun doute que le scrupuleux prélat voulait protéger nos ancêtres et leur donner un certificat de bonne moralité, qu'ils n'ont d'ailleurs

1 Ce texte est un condensé des principaux résultats (revus) du mémoire de maîtrise de Lyne Paquette, Les naissances illégitimes sur les rives du Saint-Laurent avant 1730. Université de Montréal (démographie), 1983. 202 p. Réal Bates est présentement agent de recherche au Programme de recherche en démographie historique de l'Université de Montréal.

${ }_{2}$ Jacques Henripin, La population canadienne au début du XVIIIe siècle (Paris, Presses universitaires de France, 1954), $129 \mathrm{p}$.

3 Hubert Charbonneau, Vie et mort de nos ancêtres (Montréal, Presses de l'Université de Montréal, 1975), 267 p.

4 Cyprien Tanguay, Dictionnaire généalogique des familles canadiennes (Québec, Eusèbe Sénécal), IV: 607-608. 
pas toujours mérité. ll ne faut pas trop l'en blâmer, car il ne faisait que refléter la pensée de l'Église et la mentalité de son époque.

De même l'abbé Ferland ${ }^{5}$, pour démontrer la pureté de nos or1gines, écrivait jadis ce qui suit:

A l'appui du tribut rendu à la pureté des moeurs de nos ancêtres nous citerons une autorité qui ne peut être soupçonnée de flatterie: ce sont les registres de Notre-Dame de Québec, où furent inscrits presque tous les baptêmes qui se firent dans le gouvernement de Québec, jusque vers l'année 1672. Sur six cent soixante-quatorze enfants qui furent baptisés depuis l'année 1621 inclusivement, jusqu'à l'année 1661 exclusivement, on ne compte qu'un seul enfant illégitime.

Il continuait de plus belle: «Depuis 1661 jusqu'à 1690 , on rencontre le nom d'un seul autre enfant né de parents inconnus. En sorte que dans l'espace de 69 ans, deux enfants seulement sont nés hors du légitime mariage des parents.» Or, on en dénombre 32 d'après nos calculs.

Dans la paroisse de Notre-Dame de Montréal, E.-Z. Massicotte ${ }^{6}$ ne relève pour sa part aucun baptême d'enfant né hors mariage de 1642 ì 1685 . De 1686 à 1730 , il mentionne 92 naissances illégitimes alors que nous en relevons 206 pour la même période.

S'il faut en croire ces auteurs, ainsi que certains contemporains, le libertinage est restreint dans la colonie et les problèmes de moeurs n'existent pratiquement pas. La lecture de l'ouvrage de Robert-Lionel Séguin, La vie libertine en Nouvelle-France au XVIIe siècle (1972), livre un portrait tout autre, beaucoup plus révélateur de la sexualité de nos ancêtres. Notre étude s'inscrit dans cette perspective plus large. Elle veut éclairer un point particulier de la fécondité: les naissances illégitimes du début de la colonie jusqu'à 1729 .

Les relations sexuelles hors mariage sont à l'origine du phénomène des naissances illégitimes. Bien qu'il s'agisse d'un phénomène marginal, l'étude de toute population ne saurait être complète sans l'analyse de l'illégitimité.

\section{1 - Définition des termes}

Il semble nécessaire de lever certaines ambiguïtés sur le terme de l'illégitimité et l'assimilation trop rapide que l'on a tendance à faire entre conceptions prénuptiales, enfants abandonnés, enfants trouvés et enfants illégitimes. 14

5 J.-Baptiste-Antoine Ferland, Cours d' histoire du Canada (Québec, N. S. Hardy, 1882),

6 E.-Z. Massicotte, «Comment on disposait des enfants du roi», Bulletin des recherches historiques, 37 (1931): 49-54. 
L'enfant illégitime est celui qui a été conçu et est né hors d'un mariage légitime. La notion de conception prénuptiale se réfère plutôt à l'enfant qui, né à l'intérieur du mariage, a cependant été conçu avant ${ }^{\text {' }}$. Dans un milieu fortement soumis à l'emprise de la morale chrétienne et aux pressions sociales, les mères abandonnaient parfois leur enfant. Ceci explique qu'en Nouvelle-France la presque totalité des enfants abandonnés ou trouvés étaient illégitimes. Cependant, tous les enfants illégitimes n'étaient pas abandonnés et parmi ceux qui l'ont été, se trouvaient des enfants légitimes. On retrouve peu de traces d'enfants abandonnés ou trouvés au $17 \mathrm{e}$ siècle. Le problème s'est plutôt manifesté au $18 \mathrm{e}$ siècle et surtout à partir de $1750^{8}$.

Il ne fait aucun doute que le problème de l'illégitimité est relié à un contexte religieux, social et culturel particulier, et que selon le lieu et l'époque, le problème ne se pose pas de la même façon. La société de la Nouvelle-France étant fortement marquée par l'idéologie chrétienne, la distinction entre légitimité et illégitimité est bien nette et mérite que l'on s'y arrête.

\section{2 - Sources}

Pour cette recherche, nous avons utilisé les données fournies par le Programme de recherche en démographie historique (PRDH) de l'Université de Montréal et le Dictionnaire de René Jetté ${ }^{9}$. Après avoir dépouillé les registres paroissiaux et les recensements du Québec ancien, les chercheurs du PRDH ont entrepris, à l'aide de l'ordinateur, la reconstitution de l'ensemble de la population québécoise depuis l'origine du pays ${ }^{10}$. Comme, pour l'instant, les familles n'ont pas été reconstituées au-delà de l'année 1729 , notre étude se limitera à cette date.

Les renseignements complémentaires ont été recueillis dans le Dictionnaire de René Jetté qui termine ses observations en 1730. Pour obtenir des informations sur les événements survenus après 1730, concernant le destin des illégitimes et celui de leurs parents, nous avons consulté le Dictionnaire généalogique de Cyprien Tanguay. Certes ce dernier ouvrage est lacunaire - et d'autant plus pour les illégitimes dont le destin est souvent difficile à suivre - mais les informations qu'on y trouve ne sont pas négligeables.

\footnotetext{
7 Pour connaître ce phénomène en Nouvelle-France, voir Réal Bates, Les conceptions prénuptiales dans la vallée du Saint-Laurent avant 1725. Mémoire de maîtrise, Université de Montréal (démographie), 1985, 178 p., et la deuxième partie de cette note de recherche.

8 De la Broquerie Fortier, «Les «enfants trouvés» sous les régimes français et anglais au Canada français - 1608-1850», Laval Médical, 33,7: 533.

9 René Jetté, avec la collaboration du Programme de recherche en démographique historique de l'Université de Montréal, Dictionnaire généalogique des familles du Québec des origines à 1730 (Montréal, Presses de l'Université de Montréal, 1983), 1176 p.

10 Voir Yves Landry, «Le registre de la Nouvelle-France: un outil pratique au service de la démographie historique et de l'histoire sociale», Revue d'histoire de l'Amérique française, 38,3 (hiver 1985): 423-426.
} 
La collecte de données a permis de relever 749 enfants illégitimes nés avant 1730 dont 697 ont pu être repérés par leur acte de baptême, 39 autres ont été trouvés par un acte de décès et 12 par un acte de mariage. Un seul a été retrouvé à l'aide du recensement. Nous ne possédons toutefois pas la même quantité d'informations sur tous les enfants illégitimes.

L'information relative aux parents de ces enfants n'est pas complète dans tous les cas. Au total nous connaissons les mères de 500 enfants illégitimes (soit 385 femmes différentes) et les pères de 364 de ces enfants (soit 323 hommes différents). Le tableau 1 expose ces variations.

\section{TABLEAU 1}

Répartition des enfants illégitimes et de leurs parents selon certaines caractéristiques - Canada avant 1730 .

Enfants illégitimes:

Nombre d'enfants décédés

$\mathrm{Nb}$ absolu $\quad \mathrm{Nb}$ relatif $(\%)$

Nombre d'enfants mariés

$290 \quad 38,7$

Nombre d'enfants de destin inconnu

$115 \quad 15,4$

$344 \quad 45,9$

Parents d'enfants illégitimes:

Parents inconnus

Mères inconnues - pères connus

Pères inconnus - mères connues

Parents connus

\section{1}

48

184

316

749
26,8

6,5

24,6

42,1

100,0

\section{3 - L'intensité du phénomène}

Notre première observation concerne la rareté des naissances illégitimes. Plus nombreuses entre 1700 et $1729(1,49 \%)$ qu'avant 1700 $(0,83 \%)$, elles ne forment que $1,25 \%$ des naissances de l'ensemble de la période (voir tableau 2). La France de l'époque connaissait une réalité semblable: environ 1\% de naissances illégitimes dans la France rurale, plus dans la France urbaine ${ }^{11}$. Nous constatons également que ces naissances sont deux fois plus nombreuses dans les paroisses de Québec, de Montréal et de Trois-Rivières, que nous assimilons à des villes $(1,93 \%)$, que dans les autres paroisses, essentiellement rurales $(0,87 \%)$.

11 Jacques Dupâquier, La population française aux XVIIe et XVIIIe siècles (Paris, Presses universitaires de France, 1979), 59. 
TABLEAU 2

Proportion (en \%) de naissances illégitimes par période et selon l'habitat - Canada avant 1730 -

\begin{tabular}{|c|c|c|c|c|c|c|c|c|c|c|c|}
\hline Habitat & Nombre de naissances & $\begin{array}{r}\text { Avant } \\
1680\end{array}$ & $1680-89$ & $1690-99$ & $1700-09$ & $1710-19$ & $1720-29$ & ind. & $\begin{array}{r}\text { Avant } \\
1700\end{array}$ & $1700-29$ & Total \\
\hline Rural & $\begin{array}{l}\text { illégitimes } \\
\text { totales* } \\
\text { proportion de naissances } \\
\text { illégitimes }\end{array}$ & $\begin{array}{r}17 \\
4004 \\
0,42\end{array}$ & $\begin{array}{r}20 \\
4109 \\
0,49\end{array}$ & $\begin{array}{r}40 \\
4636 \\
0,86\end{array}$ & $\begin{array}{r}90 \\
6818 \\
1,32\end{array}$ & $\begin{array}{r}83 \\
8472 \\
0,98\end{array}$ & $\begin{array}{r}102 \\
12268 \\
0,83\end{array}$ & $\begin{array}{l}- \\
-\end{array}$ & $\begin{array}{r}77 \\
12749 \\
0,60\end{array}$ & $\begin{array}{r}275 \\
27558 \\
1,00\end{array}$ & $\begin{array}{r}352 \\
40307 \\
0,87\end{array}$ \\
\hline Urbain & $\begin{array}{l}\text { illégitimes } \\
\text { totales* } \\
\text { proportion de naissances } \\
\text { illégitimes }\end{array}$ & $\begin{array}{r}22 \\
5002 \\
0,44\end{array}$ & $\begin{array}{r}18 \\
1396 \\
1,29\end{array}$ & $\begin{array}{r}57 \\
2540 \\
2,24\end{array}$ & $\begin{array}{r}73 \\
2932 \\
2,49\end{array}$ & $\begin{array}{r}78 \\
3338 \\
2,34\end{array}$ & $\begin{array}{r}127 \\
4004 \\
3,17\end{array}$ & $\begin{array}{l}- \\
-\end{array}$ & $\begin{array}{r}97 \\
9138 \\
1,06\end{array}$ & $\begin{array}{r}278 \\
10274 \\
2,70\end{array}$ & $\begin{array}{r}375 \\
19412 \\
1,93\end{array}$ \\
\hline Indéterminé & illégitimes & 1 & 3 & 4 & 7 & 1 & 4 & 2 & 8 & 12 & 22 \\
\hline Total & $\begin{array}{l}\text { illégitimes } \\
\text { totales* } \\
\text { proportion de naissances } \\
\text { illégitimes }\end{array}$ & $\begin{array}{r}40 \\
9207 \\
0,43\end{array}$ & $\begin{array}{r}41 \\
5508 \\
0,74\end{array}$ & $\begin{array}{r}101 \\
7180 \\
1,41\end{array}$ & $\begin{array}{r}170 \\
9757 \\
1,74\end{array}$ & $\begin{array}{r}162 \\
11811 \\
1,37\end{array}$ & $\begin{array}{r}233 \\
16276 \\
1,43\end{array}$ & $\begin{array}{l}2 \\
2 \\
-\end{array}$ & $\begin{array}{r}182 \\
21895 \\
0,83\end{array}$ & $\begin{array}{r}565 \\
37844 \\
1,49\end{array}$ & $\begin{array}{r}749 \\
59741 \\
1,25\end{array}$ \\
\hline
\end{tabular}

* «totales» signifie le nombre de naissances légitimes et illégitimes 
Le taux global de fécondité illégitime (nombre de naissances illégitimes pour 1000 femmes non mariées en âge de procréer) ${ }^{12}$ calculé pour l'année 1681 révèle le même phénomène, c'est-à-dire un taux plus élevé en milieu urbain, où il est de 11,2 pour mille, comparativement à 7,4 pour mille à la campagne (voir tableau 3 ).

TABLEAU 3

Taux global de fécondité illégitime en Nouvelle-France selon le type d'habitat en 1681

\begin{tabular}{lcccc}
\hline Lieu & $\begin{array}{c}\text { Nombre de } \\
\text { naissances } \\
\text { illégitimes } \\
\text { en 1680-1682 } \\
(1)\end{array}$ & $\begin{array}{c}\text { Nombre } \\
\text { annuel moyen } \\
\text { de naissances } \\
\text { illégitimes } \\
(2)\end{array}$ & $\begin{array}{c}\text { Population } \\
\text { féminine } \\
\text { non mariée de } \\
15-49 \text { ans } \\
(3)\end{array}$ & Taux \% \\
\hline Rural & 5 & 1,7 & 226 & $(2) /(3)$ \\
Urbain & 6 & 2 & 179 & 7,4 \\
TOTAL & 11 & 3,7 & 405 & 11,2 \\
\hline
\end{tabular}

1. Nombre de naissances illégitimes pour 3 années.

2. Moyenne annuelle des naissances (colonne (1)/(3)).

3. Population féminine célibataire et veuve de 15 à 49 ans tirée du recensement de 1681 .

n.b. Sont ici considérées comme urbaines les paroisses de Québec, de Montréal et de TroisRivières, et comme rurales toutes les autres.

Qu'est-ce qui explique cette plus forte proportion de naissances illégitimes en milieu urbain? La ville abrite sans doute temporairement les filles de la campagne qui portent un fruit illégitime et fuient la réprobation de leurs voisins ou espèrent accoucher subrepticement et anonymement. Mais on peut penser également que les villes, par leurs fonctions diversifiées (maritimes, commerciales, administratives et militaires) et le brassage de population qu'elles permettent, favorisent une plus grande promiscuité et un contrôle social plus difficile à exercer et donc une intensité plus grande des comportements menant à des naissances illégitimes.

Soulignons aussi l'évolution du phénomène dans le temps. Le 17e siècle ne représente que le quart de toutes les naissances illégitimes antérieures à 1730. La fréquence des naissances illégitimes augmente tout au long du 17 e siècle et se maintient à un niveau encore plus élevé au 18e siècle. Cette croissance résulte en grande partie des modifica-

\footnotetext{
12 Le taux global de fécondité illégitime est un indice nettement préférable à la proportion de naissances illégitimes comme indicateur de l'illégitimité relative. En effet, il rapporte les naissances illégitimes à la population susceptible de vivre cet événement, cependant que la proportion de naissances illégitimes est dépendante du nombre de naissances légitimes. Si, par exemple, le nombre de naissances légitimes pour une période baisse, la proportion de naissances illégitimes montrera une croissance si le nombre de ces naissances se maintient. Malheureusement le calcul de ce taux exige des recensements distribuant la population selon le sexe, l'âge et l'état matrimonial, ce qui est rare pour une population ancienne.
} 


\section{FIGURE 1}

Mouvement saisonnier des naissances légitimes et illégitimes

(Moyennes mensuelles corrigées de l'inégalité des mois)

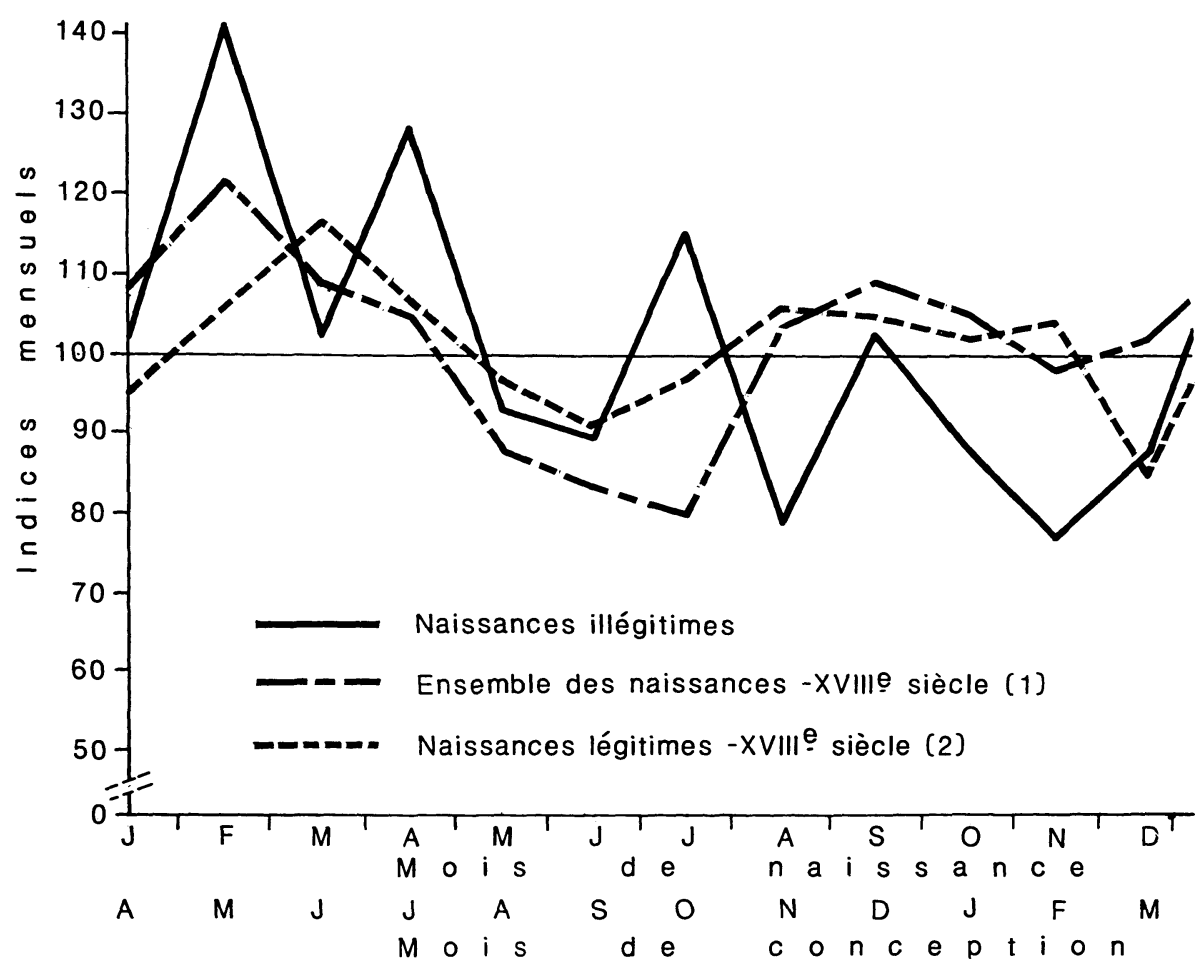

Sources: 1 R. Roy et al, "Quelques comportements des Canadiens au XVIIe siècle d'après les registres paroissiaux», Revue d'histoire de l'Amérique française, 31,1 (juin 1977): 61 .

2 J. Henripin, La population canadienne au début du XVIIle siècle (Paris, Presses universitaires de France, 1954): 42-45. 
tions survenues dans le marché matrimonial qui a été particulièrement déséquilibré au 17 e siècle. Le manque de femmes dans la colonie durant ce siècle a favorisé la précocité et l'intensité des mariages et par conséquent a réduit la natalité illégitime.

\section{4 - Le mouvement saisonnier}

L'analyse du mouvement saisonnier des naissances illégitimes met en relief le lien existant entre les conceptions (naissances moins neuf mois) et le renouveau printanier ou les travaux des champs (figure 1). Le cycle saisonnier des conceptions se caractérise par une valeur maximale au printemps avec un indice de 140 en mai. Un niveau relativement élevé se maintient durant la saison estivale. Après une baisse en août et septembre, octobre accuse une reprise. Puis l'hiver annonce une chute où les indices se retrouvent à un niveau relativement bas. Les enfants illégitimes sont donc des enfants du plein air, d'aucuns seraient tentés de dire des enfants des haies.

Le mouvement saisonnier des naissances légitimes présente certes des similitudes, mais aussi quelques différences notables. Alors que les conceptions illégitimes sont rares en janvier, les conceptions globales demeurent au-dessus de la moyenne annuelle durant ce mois. Ici également le printemps se traduit par une vigoureuse poussée de conceptions qui se poursuit jusqu'en juin. On peut comprendre la forte intensité du phénomène durant ces mois témoins du retour de la belle saison et des premiers sourires printaniers.

\section{5 - Le destin des enfants illégitimes: évaluation de la mortalité infantile}

Nous connaissons pour 362 enfants l'âge au décès (ou leur survie après 15 ans). Pour 43 autres l'âge est indéterminé (plus de trois ans, moins de 20 ans, etc.) et pour 344 le destin est inconnu. Après avoir distribué les indéterminés comme les cas connus ${ }^{13}$, nous obtenons la répartition suivante des enfants illégitimes selon leur âge au décès:

morts avant un an
morts entre un et 15 ans
morts après 15 ans
destin inconnu
TOTAL

220
66 $\quad\left(\begin{array}{r}29,4 \%) \\ 8,8 \%) \\ 119\end{array}\right.$

En supposant que tous les enfants de destin inconnu ont survécu à leur premier anniversaire nous obtiendrions un taux de mortalité infantile (minimum) de 294 pour mille. Cette hypothèse est trop optimiste pour être vraisemblable. Elle permet cependant de constater que la mor-

13 A l'exception de deux individus morts après 15 ans, les renseignements concernant ces indéterminés permettent de les répartir selon l'âge au décès des cas connus morts avant 15 ans. 
talité infantile des bâtards était des plus élevée. Dans une table de mortalité non corrigée, analogue à celle de $\mathrm{H}$. Charbonneau ${ }^{14}$, le quotient de mortalité avant un an serait de 381 pour mille, près de trois fois plus considérable que celui obtenu (135 pour mille) pour les ancêtres canadiens du $17 \mathrm{e}$ siècle.

Mais pour cerner de plus près la réalité il nous faut élaborer une table de mortalité corrigée. Celle-ci tient compte du sous-enregistrement des naissances et des actes de baptême perdus non retrouvés. Ceci ajoute 66 enfants dont 49 seraient morts avant un an ${ }^{15}$. Il s'agit ensuite de répartir les 344 enfants de destin inconnu ${ }^{16}$. Les hypothèses retenues conduisent à une mortalité infantile de 605 pour mille. Pour le $17 \mathrm{e}$ siècle, $\mathrm{H}$. Charbonneau ${ }^{17}$ a trouvé un taux corrigé de 211 pour mille et J. Henripin ${ }^{18}$, pour le $18 \mathrm{e}$ siècle, de 246 pour mille. Il resterait à voir le pourquoi de cette forte surmortalité des bâtards; mais la situation de rejet dans laquelle se trouvaient les mères, conduisant parfois à l'abandon des enfants et à la pratique de la mise en nourrice, n'y est certainement pas étrangère. A l'âge de 15 ans, seulement $22 \%$ de ces enfants auraient survécu, soit trois fois moins que les enfants légitimes.

\section{6 - Les parents des enfants illégitimes}

Après cette vue d'ensemble sur les naissances illégitimes et le destin des enfants qui en sont issus, nous examinerons maintenant la situation des parents de ces enfants, dans la mesure tout au moins où l'information recueillie le permet: il convient en effet d'interpréter les informations qui suivent avec certaines réserves puisque nous ne connaissons pas l'identité des mères de $33 \%$ des enfants illégitimes et des pères de $57 \%$ d'entre eux.

\section{1 - L'état matrimonial et l'âge des mères à l'accouchement}

Qu'en est-il de l'état matrimonial et de l'âge des mères à la naissance de ces enfants? On constate que les deux tiers d'entre elles sont célibataires alors que les veuves en représentent le quart et que pour $8 \%$ il s'agit de femmes mariées ayant commis l'adultère (voir tableau 4).

14 Hubert Charbonneau, op. cit., 121.

15 En nous appuyant sur les procédés statistiques de H. Charbonneau, op. cit., chapitre 3, nous avons obtenu 21 ondoyés décédés à moins de trois jours, 16 naissances sélectivement omises et 29 naissances perdues non retrouvées. Les deux premiers groupes correspondent à des décès très tôt après la naissance. Des 29 autres enfants, 12 , soit $40 \%$, seraient décédés avant 1 an. Hubert Charbonneau, op. cit., 122.

${ }_{16}$ Des 344 enfants au destin inconnu, 118 sont nés de parents inconnus et 54 ont une mère connue mais sont nés avant 1700 . Comme nous n'avons pas retrouvé de mariage pour ces 172 enfants, ils sont probablement tous morts avant 15 ans: nous les avons donc distribués selon l'âge au décès des enfants morts avant 15 ans. Les 172 autres dont la mère est connue et qui sont nés après 1700 ont été considérés comme décédés au même âge que les cas connus. Cela donne 223 enfants morts avant 1 an, 66 entre 1 et 15 ans et 55 après 15 ans.

17 Hubert Charbonneau, op. cit., 125.

18 Jacques Henripin, op. cit., 106. 
TABLEAU 4

Répartition des naissances d'enfants illégitimes selon l'état matrimonial de la mère à l'accouchement

- Canada avant 1730 -

\begin{tabular}{lccc}
\hline $\begin{array}{l}\text { État matrimonial } \\
\text { de la mère à } \\
\text { l'accouchement }\end{array}$ & $\begin{array}{c}\text { Nombre } \\
\text { absolu }\end{array}$ & \multicolumn{2}{c}{$\begin{array}{c}\text { Nombre relatif } \\
(\mathrm{en} \mathrm{\% )} \\
\text { Ensemble des mères } \\
(\mathrm{n}=749)\end{array}$} \\
\hline Célibataire & 331 & 66,2 & 44,2 \\
Veuve & 129 & 25,8 & 17,2 \\
Mariée & 38 & 7,6 & 5,1 \\
État indéterminé & 2 & 0,4 & 0,3 \\
Mère inconnue & 249 & - & 33,2 \\
Total & 749 & 100,0 & 100,0 \\
\hline
\end{tabular}

Pour mieux juger de cette répartition, il faudrait connaître les proportions, selon l'état matrimonial, des femmes en âge de procréation (1544 ans) dans l'ensemble de la population. En 1681 seulement 1,5\% de ces femmes sont veuves. Même si cette proportion de veuves a pu augmenter dans les décennies suivantes, il n'en reste pas moins que, proportionnellement à leur nombre, les veuves auraient plus d'enfants illégitimes que les deux autres groupes et que les femmes mariées en ont très peu (de déclarés tout au moins...).

Les mères d'enfants illégitimes ne sont pas des fillettes victimes de leur naïveté. En effet, l'âge moyen des mères à l'accouchement (tous états matrimoniaux confondus) est de 25 ans pour l'ensemble de la période. Cet âge concerne tous les accouchements d'illégitimes, peu importe le rang de ceux-ci (66 mères sur 385 auront en effet plus d'un enfant illégitime). Si on ne tient compte que de l'âge au premier accouchement, la moyenne passe à 23,3 ans.

La répartition des mères célibataires selon l'âge au premier accouchement, présentée au tableau 5 , est très révélatrice. La grande majorité ont leur enfant avant 25 ans et seulement moins de 5\% d'entre elles sont âgées de 30 ans et plus. Cela se comprend fort bien puisque plus on avance en âge, moins il y a de filles célibataires. L'âge moyen est ici de 21,6 ans. Or notons qu'entre 1621 et 1725 l'âge moyen au premier accouchement légitime pour les femmes célibataires qui se marient est également de 21,6 ans ${ }^{19}$ : on peut penser que les filles-mères ont été victimes de fausses promesses de mariage, ou, dans quelques cas, vivaient en concubinage puisqu'elles ont accouché de leur premier enfant illégitime au même âge que celles qui se sont mariées.

La situation matrimoniale particulière du $17 \mathrm{e}$ siècle tend probablement à expliquer les différences d'âge entre les périodes. L'âge au

$19 \quad$ Réal Bates, op. cit, 134. 
TABLEAU 5

Répartition des mères (célibataires) suivant l'âge à la naissance de leur premier enfant illégitime

- Canada avant 1730 -

\begin{tabular}{lrrrrrr}
\hline \multicolumn{1}{c}{ Âge } & \multicolumn{2}{c}{ Avant 1700} & \multicolumn{2}{c}{$1700-1729$} & \multicolumn{2}{c}{ Total } \\
& nb & $\%$ & nb & $\%$ & nb & $\%$ \\
\hline Avant 20 ans & 38 & 52,1 & 64 & 32,0 & 102 & 37,4 \\
$20-24$ ans & 26 & 35,6 & 78 & 39,0 & 104 & 38,1 \\
$25-29$ ans & 5 & 6,8 & 38 & 19,0 & 43 & 15,7 \\
30 ans et plus & 1 & 1,4 & 11 & 5,5 & 12 & 4,4 \\
Âge indéterminé & 3 & 4,1 & 9 & 4,5 & 12 & 4,4 \\
Total & 73 & 100,0 & 200 & 100,0 & 273 & 100,0 \\
\hline Âge moyen & \multicolumn{2}{c}{20,0} & \multicolumn{2}{c}{22,2} & & 21,6 \\
\hline
\end{tabular}

mariage étant en effet plus précoce au $17 \mathrm{e}$ siècle, les femmes ont accouché d'un enfant illégitime à un âge moins avancé.

\section{2 - Le destin matrimonial des mères}

Il importe de connaître ce que deviennent les mères des enfants illégitimes après leur accouchement. Nous avons pu suivre leur destin. La plupart des filles-mères (77\%) se marient et, par cette union, près du tiers légitiment leur progéniture en épousant le père de l'enfant. Près de la moitié se marient avec un autre homme alors que $23 \%$ demeurent célibataires. Une seule se destine à la vie religieuse (voir tableau 6).

TABLEAU 6

Destin matrimonial des mères célibataires après la naissance de leurs enfants illégitimes - Canada avant 1730 -

\begin{tabular}{lcc}
\hline Destin & Nombre absolu & Nombre relatif $(\%)$ \\
\hline -épousent le père & 80 & 29,3 \\
-épousent un autre homme & 131 & 48,0 \\
-demeurent célibataires & 62 & 22,7 \\
Total & 273 & 100,0 \\
\hline
\end{tabular}

Que 23\% de filles ayant accouché d'un enfant illégitime restent célibataires, c'est beaucoup, compte tenu de la nuptialité à cette époque. Selon Charbonneau ${ }^{20}$ «il serait étonnant que le célibat définitif féminin ait dépassé l'ordre de $10 \%$ ». Faudrait-il en conclure que la venue d'un enfant hors mariage a nui à certaines filles dans leurs chances de se marier? Pas nécessairement. Il faut prendre en compte que a) nombre

\footnotetext{
20 Hubert Charbonneau, op. cit., 158.
} 
de ces filles peuvent avoir connu une unın après 1729 (ou hors Canada) qui a échappé à Tanguay; b) que les mères célibataires forment une population sélectionnée: par définition, seules sont soumises au risque celles qui sont célibataires; celles qui ne se marieront jamais sont donc plus susceptibles de devenir mères célibataires.

\section{3 - Les pères des enfants illégitimes}

Seulement 323 pères sont connus. Mais pour certains nous ne connaissons guère plus que le nom et, parfois, la profession (soldat, commis, etc.). A toutes fins pratiques, il n'y a que 268 pères (pour 305 enfants) dont nous connaissons suffisamment de caractéristiques pour permettre l'étude statistique qui suit. C'est peu.

La plupart des pères sont célibataires $(84 \%)$. Un sur dix est marié alors que la proportion de veufs s'élève à $6 \%$. L'âge moyen de ces hommes à la naissance de l'enfant est de 29,3 ans. Quant à l'origine géographique, 99 (soit $46 \%$ ) des 215 pères dont nous connaissons l'origine sont natifs de France.

Parmi les 226 pères célibataires, 190 (84\%) se marieront: 100 (44\%) avec la mère de l'enfant, $90(40 \%)$ avec une autre femme. $16 \%$ resteront célibataires. Les mêmes réserves qu'en ce qui concerne le destin matrimonial des mères (voir point 6.2) doivent être émises ici, d'autant plus qu'on peut facilement imaginer que la proportion des pères n'épousant pas la mère de l'enfant augmenterait considérablement si nous pouvions connaître l'identité de tous ces pères inconnus.

Les pères se retrouvent surtout chez les militaires et les domestiques (61\% des 184 pères dont nous connaissons la profession). Il faut mentionner que les militaires ne manquent pas dans la colonie. A plusieurs reprises l'Église reprochera à ces hommes leur genre de vie instable et leur mauvaise conduite avec les filles:

Ayant remarqué que plusieurs jeunes gens, et particulièrement les gens de guerre, sous prétexte de rechercher des filles en mariage, se comportent de manière fort licencieuse avec les dites filles, qui se laissent abuser, sous l'espérance de les épouser, dans la persuasion qu'elles ont que les fautes et les accidents qui leur peuvent arriver en ce sujet, seront autant de motifs à leurs parents de poursuivre leurs dits mariage.... ${ }^{21}$

L'augmentation du nombre d'enfants illégitimes issus de soldats, signalée par l'évêque de Saint-Vallier, força, au 18e siècle, les autorités à permettre les mariages des soldats alors qu'ils avaient été interdits pendant un certain temps ${ }^{22}$.

\footnotetext{
21 Jean de la Croix de Saint-Vallier, Rituel du diocèse de Québec (Paris, Simon Langlois, 1703), $604 \mathrm{p}$. Emile Salone, La colonisation de la Nouvelle-
canadienne-française (Paris, Guilmoto, 1906), $467 \mathrm{p}$.
} 


\section{4 - Les récidivistes}

Nous avons tenté de mesurer quelle était la proportion de récidivistes parmi les mères et les pères d'enfants illégitimes. Il ressort clairement que la majorité de ceux-ci n'en ont eu qu'un (voir tableau 7). Il n'en reste pas moins que $17 \%$ des mères ont accouché illégitimement plus d'une fois, dont $7 \%$ au moins trois fois. Pour quelques-unes de ces femmes, les grossesses sont nombreuses. On signale ainsi pour une femme huit enfants illégitimes alors que deux autres en auront six. Il s'agit dans le premier cas du couple Thérèse Ménard et Jean Desnoyers. Mariés à la gaumine en 1709 , ce couple aura huit enfants. Le mariage ne sera réhabilité, et ne deviendra donc légitime qu'en 1724. On retrouve également 11 femmes ayant accouché de quatre enfants et plus. Ainsi, au moins $11 \%{ }^{23}$ des enfants illégitimes ne peuvent être le fruit d' «accidents». On peut sans doute évoquer l'hypothèse de la prostitution. Dans certains cas, les jugements du Conseil souverain le confirment, mais dans le cas où tous les enfants d'une récidiviste proviennent du même père, il s'agit le plus souvent de concubinage pour des couples qui se sont peut-être vu refuser le mariage par l'Église ou les parents.

\section{TABLEAU 7}

Répartition des mères et des pères selon le nombre d'enfants illégitimes qu'ils ont eus - Canada avant 1730 -

\begin{tabular}{lcccc}
\hline $\begin{array}{l}\text { Nombre } \\
\text { d'enfants }\end{array}$ & $\begin{array}{c}\text { Nombre } \\
\text { absolu }\end{array}$ & $\begin{array}{c}\text { mères } \\
\text { Nombre } \\
\text { relatif } \\
(\%)\end{array}$ & $\begin{array}{c}\text { Nombre } \\
\text { absolu }\end{array}$ & $\begin{array}{c}\text { pères } \\
\text { Nombre } \\
\text { relatif } \\
(\%)\end{array}$ \\
\hline 1 & 319 & 82,9 & 297 & 91,9 \\
2 & 39 & 10,1 & 18 & 5,6 \\
3 & 16 & 4,2 & 6 & - \\
4 & 5 & 1,3 & - & -3 \\
5 & 3 & 0,8 & 1 & - \\
6 & 2 & 0,5 & - & 0,3 \\
8 & 1 & 0,2 & 1 & 100,0 \\
\hline
\end{tabular}

Chez les pères, il existe quelques cas de récidive, mais en beaucoup moins grand nombre que chez les mères. Il faut toutefois se rappeler qu'on ignore l'identité de la plupart des pères.

23 Onze pour cent égalent le nombre d'enfants illégitimes de mères ayant accouché quatre fois et plus (55) divisé par le nombre total d'enfants (500). 


\section{CONCLUSION}

En somme, il ressort de notre étude que la natalité illégitime en Nouvelle-France a été faible au 17e siècle bien qu'elle ait augmenté progressivement au début du siècle suivant. Les proportions de naissances illégitimes sont de l'ordre de $0,8 \%$ au 17 e siècle et de $1,5 \%$ dans la période 1700-172.9. Pour expliquer cette augmentation, il reste à déterminer quelle part il faut accorder aux transformations économiques, à l'amélioration des conditions de vie, aux facteurs sociologiques et à l'évolution des mentalités. Le relèvement de l'âge au mariage demeure sans doute le facteur déterminant. Pour sa part, l'importance des naissances illégitimes dans les villes, même s'il s'agit de petites villes nullement comparables aux villes européennes de la même époque, n'est-elle pas liée au milieu urbain lui-même, à la nature des relations qu'il engendre, aux types de rapports sociaux qui y existent?

La variation des naissances illégitimes durant l'année est peut-être due aux conditions de l'environnement, telles que le climat ou les réjouissances populaires. Il semble que ces deux facteurs impriment leur marque au cycle saisonnier qui ne correspond pas à celui des naissances légitimes.

La forte surmortalité infantile des enfants illégitimes confirme leurs conditions de survie difficile.

On peut tracer une sorte de «portrait-robot» de la fille-mère canadienne aux $17 \mathrm{e}$ et $18 \mathrm{e}$ siècles. Celle-ci est canadienne de naissance et native du milieu rural. La plupart des mères d'enfants illégitimes sont célibataires et plus de $70 \%$ sont âgées de 15 à 30 ans, dont le tiers entre 20 et 24 ans. La grande majorité de ces filles se marient, et par cette union le tiers légitimeront leur progéniture en épousant le père de l'enfant.

A cette fiche descriptive de la fille-mère, on peut joindre une esquisse du portrait du père. Le plus fréquemment celui-ci est un célibataire âgé d'environ 30 ans qui exerce un métier instable.

Ces quelques éléments d'information n'épuisent certes pas le sujet de l'illégitimité en Nouvelle-France. Trop de renseignements nous manquent sur les parents des bâtards et sur le destin de ceux-ci. Il reste de plus à voir l'évolution du phénomène dans les dernières décennies du Régime français, ce que permettra bientôt la banque de données du PRDH. 